\title{
Dominio y percepción de las NIIF por los contadores públicos en Barranquilla, Colombia*
}

\author{
Domain and perception of IFRS by public accountants in Barranquilla, Colombia \\ Domínio e percepçôes das IFRS pelos contadores públicos de Barranquilla, Colombia
}

\author{
Samuel Leonidas Perez-Grau ${ }^{\text {a }}$ \\ Universidad Simón Bolivar, Colombia \\ sammy975603@gmail.com \\ ORCID: https://orcid.org/0000-0002-4105-0819
}

DOI: https://doi.org/10.11144/Javeriana.cc21.dpnc

Recibido: 12 Mayo 2019

Aceptado: 04 Octubre 2020

Publicado: 18 Diciembre 2020

\section{Resumen:}

Este artículo explica el impacto que han producido los cambios del modelo contable colombiano sobre las prácticas de los contadores públicos en Barranquilla. Estos cambios se resumen en el paso de un modelo netamente fiscalista a otro que privilegia el aspecto financiero. El objetivo es describir el dominio de las NIIF sobre las prácticas del contador barranquillero, así como la percepción de sus efectos. Esta investigación combina la encuesta y la entrevista en profundidad, trianguladas mediante documentos soportes de estudios similares. Los resultados señalan que los contadores no se sienten adecuadamente capacitados en las NIIF ni en la regulación profesional. Igualmente, los participantes cuestionan la conveniencia de estas normas, que ven como el paso a otro sistema extraño, de tradiciones foráneas y más complejo. Se concluye que el estilo sustantivista débil del marco conceptual de las NIIF relega a los contadores solamente a la deliberación técnico contable de su práctica, sin poder discutir nacionalmente posiciones de justicia, moral y sociedad.

Códigos JEL: F53, M21, M41.

Palabras clave: Contabilidad, Normas Internacionales de Información Financiera, NIIF, Consejo Técnico de la Contaduría Pública.

\section{Abstract:}

This paper explains the impacts of changes in the accounting Colombian model in Barranquilla's public accountants' practices. These changes are synthesized in the transit from a pure fiscalist model to another with only financial orientation. The aim is to describe the prevalence of the IFRS over the local accounting practices, as well as the perception of its effects. This research combines surveys and in-depth interviews, triangulated with documentary research on similar previous studies. Results show that accountants do not feel properly trained in IFRS, neither in the professional regulation. Furthermore, participants question the convenience of these regulations that they see as a strange, foreign tradition inclined, and complex system. It concludes that the weak substantivism of the IFRS's framework relegate the accountants to technically oriented decision-making practices, and exclude them from the national discussions of justice, moral and society subjects.

JEL Code: F53, M21, M41.

Keywords: Accounting, International Financial Reporting Standards, IFRS, Colombian Technical Council of Public Accounting.

\section{Resumo:}

Este artigo explica o impacto que as mudanças no modelo contábil colombiano têm produzido sobre as práticas dos contadores públicos em Barranquilla. Estas mudanças se resumem no passo de um modelo fiscalista a outro que privilegia o aspecto financeiro. O objetivo é descrever o domínio das IFRS sobre as práticas do contador local, bem como a percepção de seus efeitos. Esta pesquisa combina questionários e a entrevista em profundidade, trianguladas mediante documentos suportes de estudos semelhantes. Os resultados assinalam que os contadores não se sentem adequadamente capacitados nas IFRS nem na regulação profissional. Igualmente, os participantes questionam a conveniência destas normas, as quais vêm como o passo a outro sistema estranho, de tradições alheias e mais complexo. Conclui-se que o estilo substantivista débil do marco conceitual das IFRS relega aos contadores à deliberação técnico contábil de sua prática, sem poder discutir nacionalmente posições de justiça, moral e sociedade.

Códigos JEL: F53, M21, M41.

Notas de autor

\footnotetext{
a Autor de correspondencia. Correo electrónico: sammy975603@gmail.com
} 
Palavras-chave: Contabilidade, Normas Internacionais de Relato Financeiro, IFRS, Conselho Técnico de Contabilidade Pública.

\section{Introducción}

Desde el año 2015 Colombia trabaja en la convergencia del proceso contable a las normas internacionales. Para las pequeñas y medianas empresas, este período inició el 1 de enero de 2016 y culminó el 31 de diciembre de 2019. El propósito de esta descripción del dominio de las Normas Internacionales de Información Financiera -NIIF- y la percepción de sus efectos es el de contribuir a develar la valoración positiva a la que se ve suscrita la comunidad contable local por la adopción oficial de un nuevo sistema normativo, diversificado según agrupaciones, de condición globalizante, que replantea la práctica profesional del contador público en Colombia. En este documento se adopta como premisa que se debe superar la noción tradicional de la contabilidad como práctica e incitar a los miembros de las universidades, gremios y usuarios a divulgar los niveles complejos en que se desarrolla la disciplina, defender su autonomía y su compromiso con la sociedad.

El objetivo de este trabajo es describir el dominio de las NIIF y la percepción de sus efectos sobre la práctica profesional por parte del contador público que ejerce en la ciudad de Barranquilla, Colombia. Según García (2016, p. 8), el dominio corresponde a "la adecuada comprensión de las NIIF que debe desplegar el contador tanto para comunicarse con la gerencia como para garantizar la calidad de la información financiera”.

Sobre los efectos, Ocampo y Astudillo (2015, p. 15), conciben que "la percepción de los contadores, frente a la transformación empresarial esperada en el largo plazo tras la adopción de las NIIF, redundará en la formación de nuevos valores contables, financieros y sociales al interior de las empresas". Para Thornton y Ocasio (2008, p. 105), cabe entre sus lógicas institucionales que la percepción de los contadores públicos sobre "los efectos de la nueva normatividad sobre la práctica que les ha tocado desarrollar, aportará importantes elementos de estabilidad a los campos organizacionales de los que hacen parte, aunque estos estén siempre evolucionando y no sean estáticos".

Este estudio se desarrolla a través de una investigación mixta que combina la encuesta y la entrevista en profundidad, triangulada con investigación documental sobre estudios similares previos. La lógica conceptual del trabajo distingue las siguientes dimensiones en su desarrollo metodológico: 1) el núcleo de la actividad contable entendida normativamente; 2) la organización funcional del conjunto normativo regulador de la contabilidad; 3) el impacto de la adopción de las NIIF sobre la cultura contable; 4) el segmento de usuarios de mayor afectación por el cambio normativo; 5) las singularidades más notorias que caracterizan la nueva información contable, y 6) los referentes conceptuales que apoyan la nueva práctica.

Este artículo se estructura en cuatro secciones, además de esta introducción. En la primera sección se presenta una síntesis de la revisión de la literatura; en la segunda sección se expone la metodología; en la tercera, los resultados; y en la cuarta sección además de las conclusiones, se describen las recomendaciones pertinentes para las autoridades y empresas responsables de la aplicación de las NIIF.

\section{Revisión de la literatura}

\section{Las NIIF en Colombia}

Las Normas Internacionales de Información Financiera -NIIF- (IFRS por sus siglas en inglés), son un conjunto de normas internacionales de contabilidad promulgadas por el ente asesor mundial denominado Junta de Estándares Internacionales de Contabilidad (IASB por sus siglas en inglés) encargado de establecer los requisitos de medición, reconocimiento, presentación y revelación sobre las transacciones y actos 
contables de las empresas reflejados en los estados financieros. El IASB es una de las divisiones que junto con la Fundación de las Normas Internacionales de Información Financieras resultaron de la escisión en 2001 del Comité de Normas Internacionales de Contabilidad (IASC por sus siglas en inglés) que había sido creado en 1973 por iniciativa de un grupo de reguladores mundiales, como un ente privado e independiente, para emitir normas internacionales de información financiera dirigidas a una variedad de usuarios.

Para el año 2011, las NIIF se habían convertido en los estándares contables más reconocidos a nivel mundial. Los países como Colombia que han abrazado un proyecto de apertura y modernización económica se han apoyado en las NIIF con la finalidad de obtener confianza entre los inversionistas extranjeros e impulsar el crecimiento mediante el desarrollo de su competitividad en los mercados internacionales.

El 13 de julio de 2009, el Gobierno Nacional expidió la Ley 1314 mediante la cual la acción del Estado se dirigirá hacia la convergencia de estándares internacionales de contabilidad e información financiera en Colombia. Esta ley establece que el Consejo Técnico de la Contaduría Pública --CTCP- es un organismo permanente de organización técnica normativa contable, de información financiera y de aseguramiento de la información, adscrito al Ministerio de Comercio, Industria y Turismo. Este organismo es el encargado de proponerle a los Ministerios de Hacienda y Crédito Público y de Comercio, Industria y Turismo, la expedición de principios, normas, interpretaciones y guías de contabilidad e información financiera y de aseguramiento de la información.

\section{El debido proceso del IASB}

El proceso que sigue el IASB, es decir, la Junta de Estándares Internacionales de Contabilidad, para desarrollar una NIIF incluye cinco pasos:

1. Identificación y revisión del problema y estudio de otras normas y prácticas. En el proceso seguido por el IASB para la creación de la NIIF 13, desde 2005, la Junta de Normas de Contabilidad Financiera (FASB por sus siglas en inglés) desarrolló deliberaciones del proyecto sobre mediciones a valor razonable (Perea-Murillo, 2015, p. 764), hasta septiembre de 2006 cuando emitió la Declaración de Normas de Contabilidad Financiera, la SFAS 157, tema 820, en el que establece un marco para las mediciones a valor razonable y sus revelaciones.

2. Creación de un comité para dar consejos sobre los principales proyectos. En febrero de 2006 el IASB y el FASB acordaron un mapa de ruta y suscribieron un nuevo documento titulado: Memorándum de entendimiento 2006-2008, reafirmando su compromiso de trabajar juntos en el mejoramiento de los estándares NIIF y los USGAAP.

3. Desarrollo y publicación de un proyecto de discusión de principios que incluya los proyectos mayores. El IASB publicó en mayo de 2009 el proyecto de norma en el que propone una definición de valor razonable, un marco para medirlo y de revelaciones sobre tales mediciones (Arias y Salazar, 2012, p. 227).

4. Validación del documento de la discusión inicial y publicación de un borrador de exposición. Todo proyecto de declaración de principios, documento de discusión y borrador de exposición de un principio propuesto se publica para que sea comentado por el público. Reglamentariamente, el período usual para los comentarios es de 90 días. Las interpretaciones del proyecto se exponen con base en un período de comentarios de 60 días. En este caso, se recibieron 160 cartas de comentarios a la propuesta. El factor común de los comentarios recibidos fue la solicitud al IASB y FASB de trabajar conjuntamente en el desarrollo de estos criterios.

5. Estudio de comentarios y aprobación de un principio final. En enero de 2010 se inició formalmente el trabajo conjunto entre los dos organismos, el cual se centra en los siguientes aspectos: a) las diferencias entre los requisitos del SFAS 157 y los expuestos en el borrador de exposición del 
IASB; b) los comentarios recibidos por IASB al proyecto de discusión. Reglamentariamente, la aprobación de un principio requiere de 8 votos positivos de los 14 miembros del IASB. La NIIF 13: Medición del valor razonable fue emitida en mayo de 2011.

De lo anterior, se observa que el proceso seguido por el IASB para la adopción de nuevos estándares caracteriza un sustantivismo fuerte (Martí, 2006, p. 156) en el que, manteniendo el procedimiento democrático de la toma de decisiones, da prioridad a los valores justos, morales y sociales.

\section{El debido proceso de las NIIF en Colombia}

El procedimiento desarrollado en Colombia para adoptar las NIIF como modelo contable inicia desde la recomendación dada por la Organización Mundial de Comercio - OMC-, de la que el país hace parte, y que consiste en los siguientes hitos.

1. Revisión de las normas actuales en materia de contabilidad, auditoría, revisoría fiscal y divulgación de información, con el objeto de ajustarlas a los parámetros internacionales y para proponer al Congreso las modificaciones pertinentes, en cumplimiento de la Ley 550 de 1999 que autorizó al Gobierno Nacional en su artículo 63 llamado "Armonización de las normas contables con los usos y reglas internacionales".

2. Desarrollo y publicación de un Proyecto de Intervención Económica preparado por un Comité Técnico Institucional convocado por el Gobierno Nacional y conformado por el Ministerio de Hacienda; la DIAN; la Superintendencia Bancaria, de Valores, de Sociedades; la Contaduría General de la Nación y el Departamento Nacional de Planeación presentado a finales de 2003 a la opinión pública para su discusión, con el que se pretendía adoptar en Colombia en 2006 los estándares internacionales de Contabilidad, Auditoría y Contaduría.

3. Después de haberse recibido miles de refutaciones durante 2004 sobre si adoptar o adaptar las NIIF, el Gobierno decide suspender el proyecto. Con la Ley 1116 de 2006 que sustituiría a la Ley 550 de 1999 el Congreso otorga al Gobierno la facultad de proponer al Congreso las modificaciones necesarias para la adaptación de las NIC-NIIF cuando lo considerara prudente (Luna y Muñoz, 2011, p. 32).

4. Radicación ante la Cámara de Representantes el 10 de octubre el Proyecto de ley 165 de 2007 que otorga al Gobierno Nacional facultades de intervención para reclamar el cumplimiento forzoso en el país del nuevo tratado de internacionalización de la economía y la autoridad para ajustar la regulación contable, tramitadas dentro del marco constitucional vigente.

5. Incorporación al ordenamiento jurídico de la Ley 203 de 2008 emitida por el Senado de la República y ratificación del tratado por el Presidente de la República mediante la Ley 1314 del 13 de julio de 2009 (Marrugo y Racero, 2012, p. 72).

De acuerdo con el procedimiento anterior, puede decirse que el debido proceso colombiano para la adopción de las NIIF tipifica un sistema normativo sustantivista débil (Martí, 2006, p. 156), en el que, manteniendo ciertos contenidos mínimos de justicia, moral y sociedad, da más importancia a los procesos de deliberación y discusión, a la vez que defiende una mayor libertad al procedimiento de la toma de decisiones.

\section{Metodología}

Partiendo de la información estadística emitida por la organización gremial de Contadores Públicos de Colombia-CONPUCOL- en su Boletín 1, de agosto de 2014, con corte a 25 de junio de 2014 (Conpucol, 
2014), se encontraron registrados 197.663 contadores públicos con tarjeta profesional y 1.844 personas jurídicas con tarjeta de registro autorizadas para desarrollar actividades propias de la profesión (excepto Auditoría y Revisoría Fiscal) y, adicionalmente, retomando para esta investigación la distribución geográfica dada por la Junta Central de Contadores en tal vigencia, es posible estimar que 11.692 profesionales ejercían en el Departamento del Atlántico. El tamaño estadístico de la muestra con un límite de error muestral del 10 $\%$ y un intervalo de confianza del 99,7 \% fue estimado en 267 contadores.

Se solicitaron los listados de afiliados locales al Instituto Nacional de Contadores Públicos en Barranquilla y al Colegio de Contadores Públicos del Atlántico y con la ayuda de los estudiantes del Programa de Contaduría Pública de la Universidad Simón Bolívar se obtuvo sus localizaciones. Previa aceptación para participar en esta investigación se invitó a 267 contadores residentes en la ciudad de Barranquilla que cumplieran con un mínimo de 10 años de experiencia laboral, de los cuales, respondieron la encuesta 217 sujetos encuestados, lo que hace aceptable la deducción de inferencias con un límite de error muestral de $11 \%$ ante un intervalo de confianza del 99,7\%.

Aparte de la información general, los aspectos del desempeño actual y la experiencia laboral, el instrumento utilizado para recolectar la información utiliza una escala de Likert con las siguientes gradaciones: a) Totalmente de acuerdo, b) Algo de acuerdo, c) Indiferente y, d) En desacuerdo. El cuestionario fue sometido a revisión por parte de expertos locales en áreas de la contabilidad y la sociología. Posteriormente, se realizaron los ajustes sugeridos y se practicó una prueba piloto sobre personas con características similares a las de la población objeto de estudio, en un tamaño no superior al 10\% de la muestra. La heurística de este trabajo puede ser descrita en las siguientes tres fases.

Fase 1. Recepción y tabulación de las respuestas obtenidas. La recolección de la información culminó en enero de 2017. La información obtenida sobre las variables explicativas que han desarrollado los 217 contadores públicos respondientes acerca del nuevo modelo contable adoptado para el país se revisó, organizó y procesó mediante el uso de la estadística descriptiva. Un poco más de la tercera parte de los informantes tenían estudios de especialización. Dentro de estos, fueron identificados 37 contadores que ejercen como jefe, al menos de otro contador, a quienes les fue aplicada una entrevista en profundidad cuyos textos grabados fueron sometidos a un análisis del discurso.

El cuestionario aplicado en la fase I estuvo compuesto por seis dimensiones, cada una de las cuales comprendía cuatro preguntas o variables explicativas de la práctica profesional del contador. Los estadísticos generales que arrojó la aplicación del instrumento son: el valor promedio del puntaje total de las variables explicativas fue de 79,281 puntos, teniendo como base un puntaje máximo de 104,976 y un mínimo de 61,700. Respecto a la dispersión de los datos, la desviación estándar de 12,769 lo que podría significar una pequeña dispersión en las respuestas obtenidas en el total de la prueba, aunque esto solamente sería corroborable mediante la comparación con otros estudios.

Para medir la homogeneidad del cuestionario, se utilizó el Coeficiente de Alpha Cronbach aplicado al conjunto de respuestas de cada dimensión, como se puede ver en la tabla 1.

TABLA 1

Coeficiente Alfa Cronbach de las matrices de respuesta

\begin{tabular}{|c|c|c|c|c|c|}
\hline D1 & D2 & D3 & D4 & D5 & D6 \\
\hline 0,74 & 0,79 & 0,97 & 0,76 & 0,76 & 0,75 \\
\hline
\end{tabular}

Fuente: elaboración propia.

Si teóricamente, hasta valores de 0,70 la homogeneidad del cuestionario es aceptable, entonces el valor de 0,86 obtenido en la escala de medida del instrumento en conjunto es significativo de tener un nivel de confiabilidad adecuado.

Fase 2. Realización de entrevistas en profundidad y análisis del discurso. Las 37 entrevistas fueron acordadas previamente por vía telefónica y de correo. Resultaron más dispendiosos los acuerdos con aquellos contadores 
que ejercían como auditores por su forma de contratación laboral, que los contadores de empresa quienes casi todos podían acceder a las entrevistas en las dependencias del trabajo. El tiempo de cada entrevista osciló entre 45 y 60 minutos. Si bien existía un guión, el orden de las preguntas y la profundidad de los temas variaban según el desarrollo de cada entrevista. Siempre se trataba de obtener la máxima información posible sobre las experiencias personales de los entrevistados relacionadas con el objetivo del estudio.

Los textos grabados de las entrevistas de profundidad fueron transcritos y sometidos a un análisis del discurso con ayuda del programa Textstat 2, siguiendo las orientaciones metodológicas de la descripción "densa" de la antropología (Estrada-Mesa, 2010) acerca de la reconstrucción de una jerarquía de significados asociados a las narraciones de los participantes para explorar valores, socializaciones, reglas de interacción que permitan agrupar unidades de análisis y categorías de temas semejantes, buscando articular eventos y significados en una estructura teórica. La tabla 2 contiene las frecuencias de términos claves extraídos del análisis de los discursos obtenidos en las entrevistas de esta Fase 2.

TABLA 2

Comparativo de frecuencias de las palabras clave

\begin{tabular}{|l|c|c|c|}
\hline \multicolumn{1}{|c|}{ Categoría } & Frecuencia & \% & Dirección \\
\hline Información & 13 & 26 & $13+$ \\
\hline NIIF & 10 & 20 & $3-$ \\
\hline Normas & 8 & 16 & $3-$ \\
\hline Contable & 7 & 14 & $3-$ \\
\hline Empresas & 6 & 12 & $4-$ \\
\hline Inversores & 6 & 12 & $6-$ \\
\hline Total & 50 & 100 & \\
\hline
\end{tabular}

Fuente: elaboración propia.

La tabla 2 muestra el predominio del concepto de "Información" como reporte o estado financiero, "NIIF" o normas internacionales de información financiera y "Normas" como reglamentaciones y estatutos sobre la práctica profesional, "Contable" para referirse al contenido del trabajo profesional, el término "Empresas" hace referencia a las entidades productivas y haciendas patrimoniales, e "Inversores" quienes se dedican de oficio a financiar proyectos y emprendimientos empresariales.

Fase 3. Indagación sobre fuentes secundarias afines al tema, en bibliotecas y hemerotecas locales para establecer similitudes y coherencias entre las informaciones obtenidas mediante encuesta y las del mundo de la práctica profesional extraídas de las entrevistas en profundidad. De esta indagación documental resultaron seleccionadas las siguientes seis publicaciones de interés académico y dirigidas a la comunidad científica por los profesores: Cardona, Velandia y Giraldo (2012) relacionada con la preparación para la implementación de las NIIF de las medianas empresas del sector Dosquebradas-Pereira; Rojas y Ruiz (2016) sobre avances y dificultades del mismo proceso entre las Pymes de Villavicencio; Ferrer (2013) respecto a la convergencia a NIIF del sistema contable colombiano; Salazar-Baquero (2013) respecto a los efectos de la implementación de la NIIF para Pymes en una mediana empresa de Bogotá; Ocampo y Astudillo (2015) sobre retos y alternativas gerenciales de las NIIF en las Pymes colombianas; y García (2016) que aborda las NIIF en Colombia a la luz de los retos que deben enfrentar los contadores públicos con su implementación.

\section{Resultados}

Llegado a este punto metodológico, se precisa evitar redundancias y resaltar las relaciones de independencia entre las variables que soportan cada pregunta del cuestionario, lo que se logra con el Análisis de Componentes Principales que realiza el software Pasw SPSS 18, el cual permite el movimiento de la nube de puntos en el espacio hasta encontrar un plano de referencia sobre cuyos ejes ortogonales queden proyectadas nuevas 
variables que, en términos de vectores, hacen factible interpretar las similitudes o diferencias de variabilidades entre individuos y conjuntos, como lo es la contribución o peso respecto a la variabilidad total entre la nube original y la proyectada. Las mayores contribuciones definidas según los términos positivos obtenidos en la estimación de un primer factor y la rotación de varimax sobre los datos (Pérez-Grau, 2013) corresponden a los ítems 2, 4, 7, 9, 11, 14, 15, 16, 19, 21.

De acuerdo con este resultado, se sugiere que para futuras investigaciones se diseñe otro cuestionario con solo diez preguntas en el que se agrupen los ítems 2, 4 y 7 en una nueva dimensión que se podría denominar Legalidad; los ítems 9, 11 y 19 se podrían agrupar en la dimensión Controversia y, los ítems 14, 15, 16 y 21 podrían conformar la dimensión de Legitimidad. En tales condiciones, tomando estos nuevos datos, la medida de la homogeneidad resultó ser de 0,95. Del mismo modo, al calcular el coeficiente Alpha las nuevas agrupaciones de preguntas arrojan 0,89; 0,90 y 0,75, que se pueden considerar aceptables, buenos y excelentes, respectivamente. Como lo muestra la tabla 3, sorprende que en el primer ítem de la encuesta los contadores no ubiquen la Ley 1314 de 2009 en su carácter de norma básica.

TABLA 3

Preferencias normativas entre la totalidad de contadores

\begin{tabular}{|l|c|c|c|c|c|}
\hline $\begin{array}{l}\text { 1. La norma básica que regula la } \\
\text { práctica contable en Colombia es: }\end{array}$ & $\begin{array}{c}\text { Totalmente } \\
\text { de acuerdo } \\
(\%)\end{array}$ & $\begin{array}{c}\text { Algo de } \\
\text { acuerdo } \\
(\%)\end{array}$ & $\begin{array}{c}\text { Indiferente } \\
(\%)\end{array}$ & $\begin{array}{c}\text { En } \\
\text { desacuerdo } \\
(\%)\end{array}$ & $\begin{array}{c}\text { Total } \\
\text { (\%) }\end{array}$ \\
\hline A. El Decreto 2649 de 1993 & 47,79 & 30,23 & 21,75 & 0,23 & 100 \\
\hline B. La Ley 43 de 1990 & 63,75 & 32,00 & 4,25 & - & 100 \\
\hline C. El Decreto 2706 de 2012 & - & 79,24 & 20,76 & - & 100 \\
\hline D. La Ley 1314 de 2009 & 51,56 & 14,82 & 33,62 & - & 100 \\
\hline
\end{tabular}

Fuente: elaboración propia.

En cambio, los entrevistados que ya estaban culminando la etapa de transición, manifiestan haber tenido que ir actualizando sus conocimientos por cuenta propia, como se muestra en la tabla 4.

TABLA 4

Descripciones de actividad de los contadores

\begin{tabular}{|l|}
\hline \multicolumn{1}{|c|}{ Descripción } \\
\hline $\begin{array}{l}\text { Hay muchas fuentes de confusión como la distinción entre normas plenas y normas para } \\
\text { Pymes, lo contable y lo tributario, multilibros y multipropósito, estado de resultado y } \\
\text { otro resultado integral, entre otros. Para garantizar que las NIIF sean comprendidas por } \\
\text { los diferentes tipos de usuarios, todos requieren una formación especifica. }\end{array}$ \\
\hline $\begin{array}{l}\text { El cambio y la adaptación a un nuevo tipo de proceso contable internacionalizado choca } \\
\text { con la correspondencia entre lo profesional y lo ocupacional, circunstancias que ha } \\
\text { obligado a los contadores a reaprender sobre la marcha la lectura de los informes } \\
\text { contables y de los estados financieros. }\end{array}$ \\
\hline $\begin{array}{l}\text { Los cambios más incidentes sobre la práctica del profesional contable que trajo la nueva } \\
\text { ley colombiana tienen que ver con la estructura conceptual del proceso técnico, como son } \\
\text { el privilegio de la esencia sobre la forma, la estratificaciónde los usuarios segúnel grado } \\
\text { de aseguramiento de la información, la formalizaciónde la microempresa, la separación } \\
\text { de la contabilidad financiera y la tributaria y la separación entre las instancias de la } \\
\text { normalización técnica a cargo del CTCP y las instancias politicas a cargo de los } \\
\text { Ministerios de Hacienda y de Comercio, Industria y Turismo. }\end{array}$ \\
\hline
\end{tabular}

Fuente: elaboración propia.

Al triangular este hecho con fuentes secundarias, existe una gran coincidencia con parte de las conclusiones del trabajo de Cardona et al. (2012) relacionado con la preparación de las medianas empresas ubicadas en el sector Pereira-Dosquebradas para adoptar las NIIF, entre las que dice:

Esta investigación revela un alto nivel de desconocimiento de las normas al interior de las empresas... los responsables de la preparación de estados financieros no conocen en detalle las NIIF, lo que lleva a que la administración y la alta gerencia afronten el reto como un asunto urgente. (Cardona et al., 2012, p. 23)

Asimismo se expresan Rojas y Ruiz (2016) en su trabajo sobre avances en la preparación de las Pymes: 
Las dificultades más marcadas en el proceso de adopción y transición de las NIIF en las Pymes de Villavicencio, están relacionadas con la poca preparación que tiene el personal para afrontar el proceso, el escaso tiempo que proporcionan las entidades y los compromisos que tienen los contadores para poder capacitarse. (Rojas y Ruiz, 2016, p. 40)

Sin embargo, dada la consideración de las "muchas fuentes de confusión" y el "choque con la correspondencia entre lo profesional y lo ocupacional" que denuncian en las entrevistas los contadores como se muestra entre las descripciones de actividad vistas, se hace pertinente mencionar aquí, dos situaciones que pudieran configurarse como patrocinadoras de estas posiciones a veces negligentes y a veces reticentes de los profesionales: una, en la práctica, sigue vigente el Decreto 2649 de 1993 para aquellos asuntos no regulados por las NIIF, lo que les pudiera hacer creer que disponen de tiempos para enmiendas y, otra, en derecho, el Decreto 2548 de 2014 que remite a normas contables las normas tributarias dadas, declara en vigencia durante cuatro años más el período de aplicación de las NIIF para Pymes y empresas del Grupo 2, lo que pudiera estimular la parsimonia para adecuarse a las exigencias del nuevo sistema contable.

$\mathrm{Al}$ respecto, son destacables algunas voces de los contadores especificando situaciones en las que se vean afectados los intereses de los usuarios.

El entrevistado número 17 al justificar los retrasos de algunos de sus clientes en la entrega de la información solicitada por la Superintendencia de Sociedades afirma:

...es el mismo Estado el que propicia muchas fuentes de confusión como en el caso del Decreto 2548 de 2014 que declara en vigencia por cuatro años más el período de aplicación de las NIIF y empresas del Grupo 2, otorgando tiempo para enmiendas y remitiendo a normas contables las normas tributarias, además, ...los vacíos que ha dejado la Ley 1314, tiene uno que resolverlos según la vieja práctica apoyada por el Decreto 2649, lo que ocasiona parsimonia en la adecuación al nuevo sistema contable y un choque entre lo profesional y lo ocupacional. (Entrevistado número 17)

Por su parte el entrevistado número 24, agrega:

...el Estado debería distinguir el tipo de información que requieren las pymes para el mejoramiento de su productividad, lo que tiene más que ver con la eficiencia en el control de los recursos, el mercadeo y el informe gerencial a terceros. Realmente, los empresarios nada ganan invirtiendo altas sumas en medios tecnológicos, mientras el capital humano de las TIC insista en un lenguaje técnico que enfatiza el mercadeo y la innovación para poder penetrar en otros mercados. (Entrevistado número 24)

El segundo ítem de la encuesta sobre las funciones del Consejo Técnico se muestra en la tabla 5.

TABLA 5

Funciones del Consejo Técnico de la Contaduría Pública, CTCP

\begin{tabular}{|l|c|c|c|c|c|}
\hline $\begin{array}{l}\text { 2. El CTCP desarrolla funciones } \\
\text { orientadas a: }\end{array}$ & $\begin{array}{c}\text { Totalmente } \\
\text { de acuerdo } \\
\text { (\%) }\end{array}$ & $\begin{array}{c}\text { Algo de } \\
\text { acuerdo } \\
(\%)\end{array}$ & $\begin{array}{c}\text { Indiferente } \\
\mathbf{( \% )}\end{array}$ & $\begin{array}{c}\text { En } \\
\text { desacuerdo } \\
\text { (\%) }\end{array}$ & $\begin{array}{c}\text { Total } \\
\text { (\%) }\end{array}$ \\
\hline $\begin{array}{l}\text { A. La revisión permanente de la } \\
\text { reglamentación contable }\end{array}$ & 50,00 & 26,70 & 21,58 & 1,72 & 100 \\
\hline $\begin{array}{l}\text { B. El establecimiento de la } \\
\text { reglamentación contable para las } \\
\text { sociedades }\end{array}$ & 43,31 & 12,39 & 44,30 & - & 100 \\
\hline $\begin{array}{l}\text { C. La normalización técnica de las } \\
\text { normas contables }\end{array}$ & 49,45 & 35,49 & 15,06 & - & 100 \\
\hline $\begin{array}{l}\text { D. La vigilancia sobre el buen uso } \\
\text { de la información contable }\end{array}$ & - & 81,41 & 18,59 & & 100 \\
\hline
\end{tabular}

Fuente: elaboración propia.

Aquí, los contadores exhiben buen conocimiento sobre el CTCP como un organismo permanente de normalización técnica de normas contables, de información financiera y de aseguramiento de la información en Colombia, al tiempo que ignoran la responsabilidad de la Junta Central de Contadores para la inspección y vigilancia del ejercicio de la profesión.

Los entrevistados ven el aporte de la nueva ley en el sentido de una separación de la instancia de normalización técnica del CTCP respecto de la instancia política a cargo de los Ministerios de Hacienda y de Comercio, Industria y Turismo. Sobre esto, Ferrer (2013, p. 988) es la fuente seleccionada para enmendar la 
respuesta de los contadores por cuanto en su trabajo, que analiza el proceso de convergencia desde los factores intrínsecos al sistema contable colombiano, afirma:

La naturaleza, composición y funciones de los órganos de la profesión de que hablaba la Ley 43 de 1990, fueron modificados por la Ley 1314 de 2009 y los Decretos 691 y 1955 del 2010, para ratificar al CTCP como organismo permanente de normalización técnica de normas contables, de información financiera y de aseguramiento de la información y, a la JCC como tribunal disciplinario y órgano de registro de la población contable.

Los resultados del tercer ítem de la encuesta sobre los efectos sobre la cultura contable nacional, se exponen en la tabla 6.

TABLA 6

Afectación de la cultura contable

\begin{tabular}{|l|c|c|c|c|c|}
\hline $\begin{array}{l}\text { 3. La adopción de las NIIF afecta la } \\
\text { cultura contable al introducir: }\end{array}$ & $\begin{array}{c}\text { Totalmente } \\
\text { de acuerdo } \\
(\%)\end{array}$ & $\begin{array}{c}\text { Algo de } \\
\text { acuerdo } \\
(\%)\end{array}$ & $\begin{array}{c}\text { Indiferente } \\
(\%)\end{array}$ & $\begin{array}{c}\text { En } \\
\text { desacuerdo } \\
(\%)\end{array}$ & $\begin{array}{c}\text { Total } \\
(\%)\end{array}$ \\
\hline $\begin{array}{l}\text { A. La sistematización forzada de } \\
\text { los procesos contables }\end{array}$ & 40,39 & 15,09 & 44,52 & 100 \\
\hline $\begin{array}{l}\text { B. La injerencia de intereses } \\
\text { foráneos sobre la información } \\
\text { contable }\end{array}$ & 47,29 & 29,39 & 21,61 & 1,71 & 100 \\
\hline $\begin{array}{l}\text { C. El intervencionismo extranjero } \\
\text { sobre las cuentas nacionales }\end{array}$ & 50,82 & 33,96 & 15,22 & - & 100 \\
\hline $\begin{array}{l}\text { D. La acreditación dela prudencia } \\
\text { para el registro inmediato de las } \\
\text { pérdidas }\end{array}$ & - & 79,36 & 20,64 & - & 100 \\
\hline
\end{tabular}

Fuente: elaboración propia.

Los contadores perciben que la adopción de las NIIF afecta la cultura contable nacional junto con la llegada del intervencionismo extranjero y la introducción de prácticas extrañas. Por su parte, los entrevistados se muestran más preocupados por la mayor subjetividad y la pérdida de independencia que ahora acompaña los juicios del contador auditor. Aquí, se hace conveniente reconocer la coexistencia en el medio de otras posiciones, como lo revelan Ocampo y Astudillo (2015), quienes, en su trabajo sobre los retos y alternativas gerenciales para la implementación de la NIIF en la Pymes colombianas, dicen:

La existencia de una polaridad entre diversos factores que a favor y en contra debaten si las NIIF son convenientes para el país... de cuya consecuencia ha resultado que la Ley 1314 de 2009 es una adopción parcial de la normativa contable internacional. (Ocampo y Astudillo, 2015, p. 13)

En la tabla 7 se muestra la categorización hecha por los encuestados con respecto a la atención de la norma sobre los usuarios.

TABLA 7

Dimensión Usuarios de la Información

\begin{tabular}{|l|c|c|c|c|c|}
\hline $\begin{array}{l}\text { 4. La adopción de las NIIF ejerce } \\
\text { mayor impacto sobre el siguiente } \\
\text { segmento de usuarios: }\end{array}$ & $\begin{array}{c}\text { Totalmente } \\
\text { de acuerdo } \\
(\%)\end{array}$ & $\begin{array}{c}\text { Algo de } \\
\text { acuerdo } \\
(\%)\end{array}$ & $\begin{array}{c}\text { Indiferente } \\
(\%)\end{array}$ & $\begin{array}{c}\text { En } \\
\text { desacuerdo } \\
(\%)\end{array}$ & $\begin{array}{c}\text { Total } \\
(\%)\end{array}$ \\
\hline $\begin{array}{l}\text { A. Sobre los proveedores porque } \\
\text { pueden mantener vigente sus } \\
\text { cotizaciones }\end{array}$ & - & 85,35 & 14,65 & - & 100 \\
\hline $\begin{array}{l}\text { B. Sobre las grandes inversiones } \\
\text { al solucionar sus necesidades de } \\
\text { información }\end{array}$ & 44,81 & 39,72 & 15,47 & - & 100 \\
\hline $\begin{array}{l}\text { C. Sobre los prestamistas que } \\
\text { requieren avalúos razonables para } \\
\text { sus bienes }\end{array}$ & 57,28 & 17,79 & 23,25 & 1,68 & 100 \\
\hline $\begin{array}{l}\text { D. Sobre los clientes por su mayor } \\
\text { conocimiento del mercado }\end{array}$ & 40,45 & 20,80 & 38,75 & - & 100 \\
\hline
\end{tabular}

Fuente: elaboración propia.

Los contadores manifiestan que las NIIF favorecen a los proveedores e inversionistas. Mientras que en la tabla 8 , los entrevistados argumentan que si los informes financieros tienen que mostrar los efectos de 
las transacciones sobre los recursos y derechos satisface más la necesidad de los inversores, prestamistas y acreedores.

TABLA 8

Descripciones de valores de los contadores

\begin{tabular}{|c|}
\hline Descripción \\
\hline $\begin{array}{l}\text { El segmento más directamente favorecido con las NIIF son los usuarios financieros dadas las } \\
\text { nuevas bases de medición soportadas en estimaciones, juicios y modelos asociados a factores de } \\
\text { cantidad, tiempo e incertidumbre lo que les permiteno solo pronosticar flujos de efectivo futuros, } \\
\text { sino también evaluar el valor intrínseco de sus negocios. En la práctica, la posición de los } \\
\text { financieros circunscribe al apoyo a los valores del mercado basados en que no todas las } \\
\text { transacciones en un mercado inactivo afectan dificultades para la venta, sino por lo recuperable } \\
\text { después del pago adeudado a todos los otros grupos de interés. }\end{array}$ \\
\hline $\begin{array}{l}\text { La falta de correspondencia entre una contabilidad basada en diferentes supuestos acerca de tasas } \\
\text { de retomo, y sobrelos precios de los activos durables y, una contabilidad forense fundada sobre } \\
\text { la teoria de la evidencia positivista, amenazan seriamente la legitimidad de la profesión contable. } \\
\text { Los contadores y los auditores se han vuelto "asistentes de los usuarios de los informes } \\
\text { financieros" para que puedan asimilar la comprobación de unas representaciones arbitrarias } \\
\text { relativas a una actividad económica invisible. }\end{array}$ \\
\hline $\begin{array}{l}\text { La nueva contabilidad prioriza modelos de valoración basados en los supuestos propios de la } \\
\text { empresa acerca de precios que se asignan en el mercado. Ante esta situación riesgosa, la } \\
\text { revelación de la información sobre el proceso de valoración y la sensibilidad de los resultados de } \\
\text { la medición a los cambios en los supuestos del modelo se vuelve subjetiva y sesgada con respecto } \\
\text { a la medición. La necesidad de ampliar el espectro de los juicios obliga a la búsqueda de } \\
\text { herramientas cada vezmás avanzadas que vuelve a la práctica contable cada vez menos real. }\end{array}$ \\
\hline $\begin{array}{l}\text { Los informes financieros de propósito general no están principalmente dirigidos a las otras partes } \\
\text { como reguladores y público, ni tampoco están diseñados para mostrar el valor de la entidad, sino } \\
\text { están destinados a proporcionar información sobre los efectos de las transacciones y de otros } \\
\text { sucesos que alteran los recursos económicos y los derechos de los inversores, prestamistas y } \\
\text { acreedores y sus cambios en la entidad. La información sobre el rendimiento financiero también } \\
\text { suele indicar la medida en que los cambios en los precios del mercado o en las tasas de interés } \\
\text { han incrementado los recursos económicos y los derechos de los acreedores de la entidad, lo que, } \\
\text { de hecho, muestra una capacidad sobrevaluada para generar entradas de efectivo netas y para } \\
\text { atraer mayores financiaciones. }\end{array}$ \\
\hline
\end{tabular}

Fuente: elaboración propia.

Una tercería sobre esta manera de opinar la ofrecen Mejía, Montes y Montilla (citado por Salazar, 2013), quienes afirman:

Si se unifica el procedimiento contable a partir de la propuesta del IASB, se desconocería la multiplicidad de usuarios de la información contable y la variada gama de necesidades y de entornos existentes. Esta propuesta responde a unos usuarios en particular, a unas necesidades específicas y a un desarrollo empresarial completo. (Mejía et al., en Salazar, 2013, p. 400)

Asumiendo aquí una labor clarificadora, al tener en cuenta el reclamo de los contadores acerca de que "los informes financieros... no estén dirigidos a reguladores ni público" pudiera colegirse que existe una desconexión de los contadores locales respecto a los aspectos del análisis gerencial, estratégico y político de las empresas, como si lo es importante en la visión del modelo NIIF, sobre el entendido de que el sentido razonable de los hechos económicos se deriva de los diversos reportes de desempeño adicionales a los Estados Financieros, como refiriéndose solamente a lo financiero. Argumento que recalca Sunder (según se cita en Pacheco, Higuera, Becerra et al., 2017, p. 110):

(Bajo) el enfoque contractualista (o de agencia) de la contabilidad, a partir de la preparación de la información financiera que refleje con razonable sentido los hechos económicos, los gerentes toman las decisiones empresariales relacionadas con el desarrollo y crecimiento de los negocios y los actores del mercado las decisiones relacionadas con el ahorro, consumo e inversión. (Sunder en Pacheco et al., 2017, p. 110)

De los 37 entrevistados, cuatro tenían sus propias empresas consultoras. De hecho, puede considerarse marginal el peso de sus visiones, pero innegablemente sus descripciones ofrecen un referente complementario que especifica situaciones en las que se ven afectados sus clientes:

...la mayoría de las empresas manejan el software que llevan sus contadores y el hecho de que sea el departamento de contabilidad el único responsable de la aplicación de las NIIF y de sus ajustes contables y tributarios se debe a la falta de preparación y conocimiento de los gerentes y propietarios de las empresas. Es por esto que, una de las mayores urgencias de las empresas sea invertir en capacitación de las juntas directivas y funcionarios financieros para que aprendan a interpretar y a apoyar la aplicación de las NIIF en los nuevos estados financieros.

Por su lado, el entrevistado número 13 aclara: 
...desde el mismo Manual de las NIIF, se puede colegir que los informes financieros sirven específicamente a los inversores, prestamistas, a los otros acreedores. Estos no se hacen para mostrar el valor de la entidad que informa, ni mucho menos para ser utilizados por obreros, clientes, la vecindad o los reguladores, aunque pudieran ser útiles para ello.

Respecto al quinto punto de la encuesta, en la tabla 9, los contadores encuestados otorgan la comparabilidad, verificabilidad y oportunidad la mayor singularidad de la representación fiel de la información contable.

TABLA 9

Dimensión Singularidades de la Información

\begin{tabular}{|l|c|c|c|c|c|}
\hline $\begin{array}{l}\text { 5. La singularidad de la } \\
\text { representación fielcomo } \\
\text { caracteristica fundamental de la } \\
\text { información contable es la de ser: }\end{array}$ & $\begin{array}{c}\text { Totalmente } \\
\text { de acuerdo } \\
(\%)\end{array}$ & $\begin{array}{c}\text { Algo de } \\
\text { acuerdo } \\
(\%)\end{array}$ & $\begin{array}{c}\text { Indiferente } \\
\text { (\%) }\end{array}$ & $\begin{array}{c}\text { En } \\
\text { desacuerdo } \\
(\%)\end{array}$ & $\begin{array}{l}\text { Total } \\
\text { (\%) }\end{array}$ \\
\hline $\begin{array}{l}\text { A. Completa, neutral y libre de } \\
\text { error }\end{array}$ & 43,38 & 12,37 & 44,25 & - & 100 \\
\hline $\begin{array}{l}\text { B. Influyente, de valor predictivo } \\
\text { y de valor confirmatorio }\end{array}$ & - & 81,45 & 18,55 & - & 100 \\
\hline $\begin{array}{l}\text { C. Comparable, verificable y } \\
\text { oportuna }\end{array}$ & 59,53 & 25,62 & 14,85 & - & 100 \\
\hline $\begin{array}{l}\text { D. Comprensible, neutray } \\
\text { verificable }\end{array}$ & 49,80 & 26,93 & 21,56 & 1,71 & 100 \\
\hline
\end{tabular}

Fuente: elaboración propia.

Como se muestra en la tabla 10, los entrevistados piensan que el modelo contable de las NIIF es extraño a la práctica contable local pues la subjetividad acompaña a los juicios y si hay que advertir sobre las incertidumbres del mismo, entonces se estaría buscando con ello una validación del concepto antes de emitirlo.

TABLA 10

Descripciones de posición de los contadores

\begin{tabular}{|c|}
\hline Descripción \\
\hline $\begin{array}{l}\text { La nueva normatividad ha llegado a través de un proceso de adaptación a un modelo ajeno, lo } \\
\text { que ha traido consigo una sobreestimación de los ingresos futuros y una subestimación de los } \\
\text { ingresos netos. Ha surgido una demanda creciente sobre unas revelaciones de la utilidad que } \\
\text { no pasa por los resultados, como en el caso de las relacionadas con las contabilizaciones } \\
\text { distribuidas homogéneamente en el tiempoy, con el riesgo asociado a los títulos valores que } \\
\text { no sería realizable hasta su renegociación, lo que involucra a los contadores en un } \\
\text { conservadurismo de las ganancias pues al final aparecerian las pérdidas. }\end{array}$ \\
\hline $\begin{array}{l}\text { Bajo Representación fiel, información "completa" significa la existencia de caracteristicas de } \\
\text { similitud con las de otra entidad que informa antes de poder referenciar algún grado de } \\
\text { comparabilidad respecto a cierto fenómeno económico pertinente; una información se hace } \\
\text { "neutral" si para quien informa representa de manera fidedigna los eventos económicos, } \\
\text { aunque haya sido generada por estimaciones. Un reporte estaría "libre de error" si va } \\
\text { acompañado de advertencias sobre las incertidumbres que afectan de forma significativa las } \\
\text { estimaciones. Se trata de un esquema subjetivo en búsqueda de validación colectiva al modo } \\
\text { de la construcción de un consenso antes de comunicar su existencia a terceros. }\end{array}$ \\
\hline
\end{tabular}

Fuente: elaboración propia.

En este punto ha sido necesario confrontar estas dos posiciones con sendas fuentes secundarias. Por una parte, afirma Ocampo y Astudillo (2015, p. 13) que "la percepción de extrañeza del modelo contable de las NIIF ha sido un aspecto repetidamente señalado en el ambiente profesoral y académico". Por la otra parte, Ferrer (2013, p. 1001) dice:

Se requiere trabajar en la relación contabilidad-sociedad, en los cambios sociales que traerá la simbiosis de las prácticas locales empresariales y el nuevo modelo a aplicar, lo cual puede ser canalizado a través del debido proceso establecido por el IASB y, en caso de que su incorporación no sea viable, se puedan establecer excepciones locales que potencien las actividades de determinado sector.

La posición sentida de los contadores al especificar situaciones en las que se vean afectados los intereses de los usuarios pudiera ser indicativo de grados de comprensión y aceptabilidad. Al respecto, el entrevistado número 31 expresa: 
Las NIIF parecen ser de libre interpretación y se corre el riesgo de que los contadores de las empresas manipulen las cifras a su amaño. (Entrevistado número 31)

El entrevistado 6 dice:

...las NIIF responden a una tendencia mundial y como tal, los argumentos que la sustentan se muestran más publicitarios que comprobables “...las NIIF ayudan a las grandes firmas a proporcionar información útil para los inversores nacionales en los mercados de valores o para la conformación de alianzas o compraventa entre firmas que interesan a los inversionistas extranjeros. (Entrevistado número 6)

El entrevistado 27, afirma:

...las NIIF configuran un proceso complicado, dispendioso e irreal. Por un lado, tenemos el valor razonable que puede presentar inconsistencias relacionadas con la fiabilidad de las cifras, producto del uso de bases estadísticas que no están relacionadas con la realidad económica y, por el otro, la descripción fiel para explicar las incertidumbres que afectan de forma significativa las estimaciones. (Entrevistado número 27)

Finalmente, la tabla 11 se refiere al sexto punto de la encuesta acerca de la percepción de utilidad del marco conceptual. Al respecto, los contadores ven la utilidad del marco conceptual para elaborar, sistematizar y regular la información contable.

TABLA 11

Dimensión Marco Conceptual

\begin{tabular}{|l|c|c|c|c|c|}
\hline $\begin{array}{l}\text { 6. La principal utilización del } \\
\text { marco conceptualsobre las } \\
\text { normas contables del pais es: }\end{array}$ & $\begin{array}{c}\text { Totalmente } \\
\text { de acuerdo } \\
(\%)\end{array}$ & $\begin{array}{c}\text { Algo de } \\
\text { acuerdo } \\
\text { (\%) }\end{array}$ & $\begin{array}{c}\text { Indiferente } \\
\text { (\%) }\end{array}$ & $\begin{array}{c}\text { En } \\
\text { desacuerdo } \\
(\%)\end{array}$ & $\begin{array}{c}\text { Total } \\
\text { (\%) }\end{array}$ \\
\hline $\begin{array}{l}\text { A. Para una mejor elaboración de } \\
\text { la información contable }\end{array}$ & 56,08 & 29,01 & 14,91 & - & 100 \\
\hline $\begin{array}{l}\text { B. Para proporcionar un referente } \\
\text { estable al ejercicio de las } \\
\text { facultades regulatorias de las } \\
\text { autoridades }\end{array}$ & 52,61 & 24,32 & 21,37 & 1,70 & 100 \\
\hline $\begin{array}{l}\text { C. Para operacionalizar la } \\
\text { sistematización de las } \\
\text { transacciones contables }\end{array}$ & - & 83,15 & 16,85 & - & 100 \\
\hline $\begin{array}{l}\text { D. Para regular la conformación } \\
\text { de los grupos segin niveles de } \\
\text { cumplimiento de las normas }\end{array}$ & 44,67 & 13,25 & 42,08 & - & 100 \\
\hline
\end{tabular}

Fuente: elaboración propia.

Los entrevistados, por su parte, disienten sobre que la nueva ley use adecuadamente el término convergencia que correspondería a un proceso de discusión de los asuntos sustantivos para llegar a un acuerdo y consideran al marco conceptual como dejando que esto hagan los usuarios, pero sobre los asuntos procedimentales, situación que propicia una percepción abusiva de la legalidad en detrimento de la aceptación como legitimidad tal como lo muestra la tabla 12.

TABLA 12

Descripciones de relación de los contadores

\begin{tabular}{|c|}
\hline Descripción \\
\hline $\begin{array}{l}\text { En la versión } 2010 \text { del Marco Conceptual, aparece sustituida la hipótesis fundamental } \\
\text { del Devengo, como era considerada en la versión anterior, en una información } \\
\text { descriptiva de los efectos de las transacciones. La fiabilidad que aparecía entre } \\
\text { características cualitativas de los estados financieros pasa ahora a característica } \\
\text { fundamental con el nombre de Representación fiel junto con las de Relevancia y } \\
\text { Materialidad, al tiempoque pasan a características de mejora, las de Comparabilidad, } \\
\text { Oportunidad y Comprensibilidad. A su vez, estos cambios obligan al cambio de } \\
\text { apreciación sobre la información contable. }\end{array}$ \\
\hline $\begin{array}{l}\text { El marco conceptual para la preparación y presentación de estados financieros, es la } \\
\text { parte compensatoria a un sustantivismo fuertepreviamente definido a la instauración de } \\
\text { la norma para que los contadores den mayor importancia a la deliberación y discusión } \\
\text { sobre la conversión del acto económico en acto contable, defendiendo una mayor } \\
\text { libertad para pactar con los usuarios de la información las diferencias de interpretación } \\
\text { de las normas antes de llegar al procedimiento de la toma de decisiones. }\end{array}$ \\
\hline
\end{tabular}


El entrevistado 10 dice:

...realmente el Marco conceptual desatiende los intereses de los otros tipos de usuarios de la información contable distintos al inversor...tampoco quiere ver la riqueza más allá de su posibilidad reproductora del capital, ni menos pretender el control de los resultados más allá de la predicción financiera..., definitivamente, no hay allí preocupación para que sean incluidos los asuntos sociales, de gobernanza y ambientales como parte del informe gerencial anual.

El entrevistado 19 afirma:

...el Marco conceptual en sí no tiene la fuerza de una norma contable pues ante algún caso eventual de diferencias de interpretación los procesos de consenso no hacen obligatoria su aplicación.

La contrastación de estas respuestas la ofrece la opinión dada por el experto internacional Waldo Moticorena (como lo cita Rojas y Ruiz, 2016), quien, refiriéndose a los fundamentos en la planeación, dirección y ejecución de cualquier proceso de implementación de NIIF, manifiesta:

Lo esencial es la apropiación del marco conceptual, el cual es casi como un espejo de lo contenido en el Decreto 2649 de 1993, desafortunadamente al igual que muchos otros países, los contadores colombianos se acostumbraron a seguir las reglas de la Dirección de Impuestos y Aduanas Nacionales y de los otros entes reguladores para el tratamiento de las transacciones económicas e inconscientemente ignoraron los preceptos del Marco Conceptual. (Waldo Moticorena en Rojas y Ruiz, 2016, p. 6)

Los aspectos que pudieran limitar la cobertura del cumplimiento de objetivos se refieren a:

1. La falta de actualización del censo de profesionales inscritos en las asociaciones gremiales;

2. La heterogeneidad de los efectos de las normas sobre los tipos de cargos que desempeñan los contadores, ya sean empleados, auditores, tributaristas, entre otros;

3. La etapa en el proceso de adopción de las NIIF en que se encuentren los profesionales.

\section{Conclusiones}

Esta investigación concluye que, principiando el año de 2017, a los contadores públicos en ejercicio en la ciudad de Barranquilla, les asiste buena voluntad para capacitarse, en el caso de contar con el apoyo estatal o de la empresa, aunque el desigual ofrecimiento de esta oportunidad, en cuanto a NIIF, conduce a niveles diferentes de respuestas en los casos examinados, para poder garantizar la correcta y consciente aplicación de estas normas en las empresas a las cuales prestan sus servicios profesionales.

La población estudiada de contadores públicos que ejercen su profesión en Barranquilla es consciente de la realidad perentoria de aplicación de las NIIF lo que les exige la apropiación precisa de unas responsabilidades éticas, legales y civiles establecidas para asegurar el cumplimiento eficiente de sus obligaciones, sin embargo, entre ellos coexiste una proporción sensible que aún siguen prácticas contables de interpretación tradicional.

Los contadores que participaron en el estudio manifiestan preocupaciones culturales de naturaleza política y técnica, relacionadas con la profesión. En lo político, persiste en ellos una idea de rechazo al origen foráneo de las NIIF. En lo técnico, sostienen la idea de que las prácticas contables relacionadas con la valoración de los hechos económicos, el mayor detalle de las revelaciones contables, el predominio de los sistemas de información, la segmentación de la información, la valoración del riesgo y la contabilización de cobertura, son complejas y requieren un tiempo de uso y aplicación para poder dominarlos.

Los contadores entrevistados perciben que las NIIF están hechas principalmente para satisfacer las necesidades de los inversores y acreedores, en contrario al modo de la anterior ley local que establecía mecanismos de estratificación para los pequeños y medianos empresarios, lo que de por sí ha determinado el carácter parcial de la Ley 1314 de 2009 respecto a la concepción contable internacional. 
Los contadores resienten el paso del paradigma de la maximización de las utilidades al de la utilidad de la información para la toma de decisiones, basados en la introducción de conceptos exóticos como los de la representación fiel y la confiabilidad, que buscan reflejar la correcta estimación e incertidumbre que rodea a los eventos económicos de la entidad y, en los de comparabilidad y verificabilidad con los que se pretende establecer la identificación y alcance de las similitudes y diferencias entre partidas contables.

En particular, los contadores de Barranquilla censuran el modo sustantivista débil (Martí, 2006, p. 156) del proceso colombiano de adaptación de las NIIF que deja a los profesionales contables la sola posibilidad de intervención en los procesos deliberativos y de discusión sobre la técnica contable y les niega el derecho de ejercer un normativismo fuerte, como es aplicado por el IASB en el debido proceso seguido para la formulación o la modificación de normas, que permita discutir nacionalmente posiciones de justicia, moral y sociedad.

Para futuras investigaciones se sugiere diseñar otro cuestionario con menos preguntas que el utilizado en la presente investigación, y agrupar los ítems en dimensiones, que podrían denominarse Legalidad, Controversia y Legitimidad.

Adicionalmente, con el deseo de acrecentar en algo el entendimiento de las condiciones y alternativas de mejoramiento con que aún puede contar la práctica contable profesional, se relacionan las siguientes recomendaciones dirigidas a las autoridades técnicas, de regulación y de educación del país contable:

- Propiciar encuentros académicos y gremiales entre el CTCP y los grupos de contadores, universidades y de los gremios de la producción para verificar las experiencias, dificultades y propuestas de flexibilidad de las normas, que lleve a la unificación de criterios de interpretación que las normas generan entre los diversos niveles de aplicación.

- Proponer procesos educativos contables de gran cubrimiento nacional que trate no solamente aspectos de la técnica contable sino su relación con todos los otros subsistemas de la organización empresarial que ayuden a un trabajo conjunto como lo concibe la contabilidad internacional.

- Fomentar la investigación sobre incidencias mutuas que tiene lugar entre el sistema contable y la sociedad con motivo de la aplicación de las NIIF, que pueda conducir al planteamiento de propuestas de ajustes a las normas, procurando satisfacer las necesidades específicas de los diversos sectores de la actividad económica nacional.

\section{Agradecimientos}

Este es un artículo de investigación científica y tecnológica realizado durante los años de 2016-2017, derivado del proyecto Determinación del impacto en el desempeño profesional del contador público por la aplicación de las NIIF, financiado por la Universidad Simón Bolívar iniciado desde febrero de 2013 bajo el código interno 03010030213

\section{Referencias}

Arias, M., \& Salazar, E. (2012). Efecto del debido proceso en la formulación de la NIIF 13. Revista de la Facultad de Ciencias Económicas de la Universidad Nueva Granada, 20(1), 207-229. https://doi.org/10.18359/rfce.2193

Cardona, J., Velandia, M., \& Giraldo, J. (2012). Nivel de preparación de medianas empresas del sector industrial Pereira - Dosquebradas para adoptar normas internacionales de contabilidad. Aglala, 3(1), 11-26. https://doi.org/10.2 $2519 / 22157360.877$

Compucol (2014). Boletín 1 Actualícese. Recuperado de: https://actualicese.com/actualidad/2006/08/09/colegio-d e-contadores-boletin-001/ 
Estrada-Mesa, A. (2010). Recursos críticos interpretativos para la psicología social. Revista colombiana de psicología, 19(2), 261-270. URL: https://www.researchgate.net/publication/49609212_RECURSOS_CRITICO-INTE RPRETATIVOS_PARA_LA_PSICOLOGIA_SOCIAL

Ferrer, A. (2013). Análisis del proceso de convergencia a normas internacionales de contabilidad e información financiera desde los factores intrínsecos al sistema contable colombiano. Cuadernos de contabilidad, 14(36), 971-1007. file://C:/Users/sammy/Downloads/7910-Texto\%20del\%20art \%C3\%ADculo-30067-1-10-20140319.pdf

García, D. (2016). La importancia de la NIIF en Colombia: un reto para los contadores públicos. https://repository.uni militar.edu.co/bitstream/10654/15262/1/GarciaLopezDarwinJesus2016.pdf

López, J. (2009). El concepto de legitimidad en perspectiva histórica. Cuadernos Electrónicos de Filosofía del Derecho,18(1), 153-168. https://doi.org/10.7203/CEFD.18.116

Luna, J., \& Muñoz, L. (2011). Colombia: hacia la adopción y aplicación de las NIIF y su importancia. Adversia, 8(1), 26-43. https://revistas.udea.edu.co/index.php/adversia/article/view/10954

Martí, J. L. (2006). La república deliberativa: una teoría de la democracia. Barcelona: Marcial Pons. https://www.mar cialpons.es/libros/la-republica-deliberativa/9788497683838/

Marrugo, C., \& Racero A. (2012). Análisis de la estandarización de las NIIF en Colombia. Tesis de pregrado inédita. Facultad de derecho y ciencias políticas, Universidad de Cartagena.

Ocampo, P., \& Astudillo, R. (2015). Retos y alternativas gerenciales en la implementación de las NIC-NIIF en las Pymes colombianas. Magazin empresarial, 11(26), 11-19. https://repository.usc.edu.co/bitstream/20.500.12421/2486/1/Retos\%20y\%20alternativas\%20gerenci ales\%20en\%20la\%20implementaci\%C3\%B3n\%20de\%20las\%20NIC-NIIF\%20en\%20las\%20pymes\%20colo mbianas.pdf

Pacheco, G., Higuera, V., Becerra, J., Aguirre, J., \& Gonzalez, R. (2017). Paradigmas emergentes de la contabilidad y las tendencias investigativas de alto impacto. Revista Lasallista de Investigación, 4(1), 103-111. https://doi.org /10.22507/rli.v14n1a9

Perea-Murillo, S. (2015). Perspectiva crítica del valor razonable en el marco de la crisis financiera. Cuadernos de Contabilidad, 16(42).761-779. https://revistas.javeriana.edu.co/index.php/cuacont/article/view/17392

Pérez-Grau, S. (2013). Selección de índices financieros mediante técnicas estadísticas del análisis multivariante. Entramado, 9(1), 118-140. https://revistas.unilibre.edu.co/index.php/entramado/article/view/3448

Rojas, M., \& Ruiz, M. (2016). Avances, limitaciones y dificultades en la preparación de las Pymes de Villavicencio en su proceso de transición. https://docplayer.es/60002493-Villavicencio-en-su-proceso-de-transicion-resumen-la-im plementacion-de-las-normas-internacionales-en-colombia-se-convirtio-en-una.html\#show_full_text

Salazar-Baquero, E. (2013). Efectos de la implementación de la NIIF para las Pymes en una mediana empresa ubicada en la ciudad de Bogotá. Cuadernos de Contabilidad, 14(35), 95-414. https://revistas.javeriana.edu.co/index.ph $\mathrm{p} /$ cuacont/article/view/7097

Thornton, P., \& Ocasio, W. (2008). Institutional logics. The Sage handbook of organizational institutionalism. London: Sage Publications.

\section{Notas}

* Artículo de investigación.

\section{Licencia Creative Commons CC BY 4.0}

Para citar este artículo: Perez-Grau, S. L. (2020). Dominio y percepción de las NIIF por los contadores públicos en Barranquilla, Colombia. Cuadernos de Contabilidad, 21. https://doi.org/10.11144/Javeriana.c c21.dpnc 